\title{
Definiendo la hipertextualidad. Análisis cuantitativo y cualitativo de la evolución del concepto
}

\section{Defining hypertextuality. Quantitative and qualitative analysis of the evolution of the concept}

\author{
Félix Arias-Robles \\ Profesor Asociado del Grado en Periodismo y \\ Subdirector del Máster en Innovación en Periodismo \\ (Universidad Miguel Hernández) \\ José Alberto García-Avilés \\ Profesor Titular de Periodismo \\ (Universidad Miguel Hernández)
}

Fecha de recepción: 15 de julio de 2016

Fecha de revisión: 5 de septiembre de 2016

Para citar este artículo: Arias-Robles, F. y García-Avilés, J.A. (2016):

Definiendo la hipertextualidad. Análisis cuantitativo y cualitativo de la evolución del concepto, Icono 14, volumen 14 (2), pp. 48-68. doi: 10.7195/ ri14.v24i2.995 


\section{Resumen}

Pese a las múltiples referencias a la hipertextualidad, todavía existen confusiones sobre su significado. Ahora que cumple medio siglo, resulta pertinente recopilar y aclarar el significado de un término todavía esencial para entender la naturaleza de los contenidos digitales. Por eso, esta investigación realiza un análisis documental y de contenido de un conjunto de 155 referencias en 136 trabajos seleccionados a partir de una muestra inicial de 4.750. El estudio de variables como el año de publicación, el tipo de mención o los elementos presentes en las definiciones permite estudiar la evolución, desviaciones y, sobre todo, puntos en común. Este proceso pone de manifiesto la juventud de la mayoría de las referencias conceptuales, su estabilidad en el tiempo y la tendencia a la concreción. Pero, sobre todo, demuestra la necesidad de incluir en su definición elementos como estructura, contenido multimedia, interfaz digital y, especialmente, enlace y usuario.

Palabras clave: Hipertextualidad - Terminología - Análisis documental - Análisis de contenido - Contenidos digitales - Enlace - Usuario

\section{Abstract}

Despite the numerous references to the concept of hypertext, there are still confusion and disagreement about its meaning. Now that it is fifty years old, it is appropriate to gather and clarify the meaning of a still essential to understanding the nature of digital content term. That is why this research is based on a document and content analysis of a set of 155 references on a corpus of 136 works selected from an initial sample of 4,750. The study of variables such as the year of publication, the type of indication or the elements in these proposals allow for study the evolution, deviations and, above all, the common ground. This process reveals the youth of most conceptual references, their stability over time and their tendency toward concreteness. But above all it demonstrates the necessity to include in their definition elements such as structure, multimedia content, digital interface and, especially, link and user.

Key Words: Hypertextuality - Terminology - Document analysis - Content analysis Digital content - Link - User 


\section{Introducción}

Cuesta encontrar un término tan controvertido como la hipertextualidad. La constante transformación tecnológica ha generado, encumbrado y enterrado decenas de conceptos. Algunos tan peregrinos que apenas se recuerdan. Otros tan sonoros y singulares que resonaron con estrépitos tras unos pocos discursos. Pero pocos han durado tantos años y han despertado tanto interés, entusiasmo, descrédito y nostalgia como el que da nombre a la interconexión de contenidos mediante enlaces.

El problema de la hipertextualidad, por lo tanto, más allá de sobreponerse al capricho de las modas terminológicas, radica en su dispersión conceptual. La mayor parte de las reflexiones sobre la naturaleza de este fenómeno poseen nexos en común, pero también existen matices considerables e importantes desviaciones.

Por eso, este trabajo aspira a satisfacer la necesidad de reunir, sistematizar y destilar este concepto. Para ello, tras recopilar sus menciones en la bibliografía académica de campos como la informática, la documentación, las humanidades y el periodismo, se analiza la evolución de sus diversas tendencias, sus puntos en común y sus divergencias.

Las palabras no dejan de ser simples convenciones. Nada más lejos de esta investigación, por lo tanto, que obsesionarse con una de ellas. Entre otras cuestiones, porque probablemente pasará a la historia como el resto:

Sólo algunos viejecitos nostálgicos (hoy cibermodernos, cibernautas, ciberpijos) aburrirán a sus nietos con términos que a las criaturitas les sonarán a chino: interactivo, digital, multimedia, libro electrónico, correo electrónico, cibernauta, internauta... ¿Para qué decir, por ejemplo, «digital interactivo» si todas las comunicaciones serán digitales e interactivas? Si ya nadie dice cine sonoro en color y en estéreo, ¿por qué se iba a decir a mediados del siglo XXI web televisión digital interactiva? (Moreno, 2002: 24-25)

Pero mientras tanto, al menos, conviene entender su verdadero significado, que en el fondo no es más que el conjunto de las definiciones que sobre ella se han 


\section{INNOVACIÓN TEÓRICA}

formulado. De este modo, al menos mientras se emplee o se lea, será más fácil comprender a qué hace referencia la palabra "hipertextualidad".

\section{Método}

Esta investigación se basa en la combinación de las técnicas propias del análisis documental y el de contenido. En primer lugar, la catalogación y la categorización de los documentos que conforman el corpus de esta investigación (Andréu, 2001: 9) facilita el estudio posterior de las definiciones (Dulzaides y Molina, 2004) desde un punto de vista lingüístico (Peña y Pirela, 2007: 55) y formal (Clauso, 1993: 11).

En la segunda metodología empleada, además, se desarrollan sus dos variantes: la cuantitativa y la cualitativa. La parametrización de los resultados obtenidos permite formular, a partir de datos concretos, "inferencias reproducibles y válidas que puedan aplicarse a su contexto" (Krippendorff, 1990: 28). El estudio en detalle de algunos de los casos estudiados, por su parte, permite profundizar en los matices de una parte de las definiciones (Cáceres, 2003: 53-55).

La recopilación del material se realizó en tres fases: en la primera, se llevó a cabo la búsqueda de los términos "hipertextualidad" e "hipertexto", en castellano y en inglés, en nueve de las principales bases de datos científicas: ISOC (CSIC), Scopus, ScienceDirect, ProQuest, Web of knowledge, Emerald Insight, IEEE, Springer y Wiley.

En segundo lugar, se consultaron las principales revistas académicas y las actas de congresos que aparecieron como resultado en la búsqueda inicial o que se consideraron cercanas al tema de estudio. De este modo, se prestó una especial atención a publicaciones de editoriales internacionales como las de SAGE o Taylor \& Francis, así como a las editadas en las principales universidades del ámbito hispano y anglosajón.

Por último, se llevó a cabo un proceso de rastreo entre las citas y referencias bibliográficas presentes en los trabajos localizados. De este modo, se detectaron otros trabajos, generalmente monográficos, manuales o artículos de divulgación que habían quedado fuera de las principales editoriales o bases de datos. 
En este punto, hay que resaltar algunas limitaciones que probablemente expliquen, al menos en parte, la reducción de los casos detectados en los últimos años. Durante este tiempo, posiblemente se realizaron menciones que no se pudieron detectar porque, dado que la recopilación inicial se realizó a finales de 2014, algunas investigaciones no aparecerían en las bases de datos consultados. Esto también explica que, como se podría intuir, las publicaciones más recientes no aparezcan en las referencias bibliográficas que complementaron esta búsqueda.

Una vez recopilado el corpus bibliográfico, se llevó a cabo una preselección compuesta por un total de 4.750 textos: 3.320 artículos, 790 libros o capítulos y 430 comunicaciones y 210 artículos de divulgación. Entre todos estos, posteriormente se escogieron sólo aquellos que hicieran referencia directa al concepto de hipertextualidad más allá de su descripción técnica -como parte del Hypertext Transfer Protocol (HTTP)- o su mención como característica de los contenidos digitales.

Finalmente, tras descartar las repeticiones o las citas secundarias, esta investigación se basó en un total de 155 menciones a este término en un intervalo temporal de 49 años: desde 1965 hasta 2014. Esto se traduce en una media de alrededor de 3 menciones al año, aunque como luego se explica, distribuidas de manera irregular. Se optó por contabilizar más de una propuesta en un único trabajo si aquella se desarrollaba de manera independiente y poseía una entidad suficiente. De este modo, se analizó un total de 136 trabajos distribuidos de la siguiente manera: 81 artículos científicos, 36 libros capítulos de libros, 17 comunicaciones y 2 textos divulgativos.

Tras un análisis sobre su distribución a lo largo del tiempo, estos datos se analizaron en función de su tipología. Se consideraron definiciones aquellas explicaciones explícitas sobre el significado de la hipertextualidad; metáforas, las referencias en las que este fenómeno se exponía mediante una construcción figurada; metadefiniciones, las disquisiciones en las que se recopilaban o criticaban otras propuestas teóricas previas; y menciones etimológicas, las que simplemente hacían referencia a una de las dos partes que componen esta palabra: "hiper" y "texto".

A continuación, se profundizó en las definiciones, las referencias más directas a este concepto y, por lo tanto, las más pertinentes para esta investigación. Para ello, 


\section{INNOVACIÓN TEÓRICA}

se dividieron entre las que tenían un cariz descriptivo, con alusiones claras a objetos concretos o medibles; las abstractas, con discusiones más etéreas e ideas menos tangibles; y las etimológicas, en las que esos componentes de la hipertextualidad ejercían un papel determinante en su descripción. Como es lógico, en muchos casos conviven más de uno de estos subtipos, pero se optó siempre por escoger el predominante.

Finalmente, se extrajeron los conceptos clave de estas definiciones mediante un doble procedimiento: la anotación de los términos más relevantes y la utilización de un software de análisis de contenido cualitativo. Para facilitar su análisis, se llevó a cabo un proceso de homogenización de los términos que hacían referencia a una misma realidad, con objeto de facilitar así un análisis más comprensible. Y para clarificar la visualización, sólo se recogieron en un gráfico aquellos conceptos que aparecieron en más de una propuesta.

\section{Desarrollo}

\subsection{Evolución temporal de las referencias}

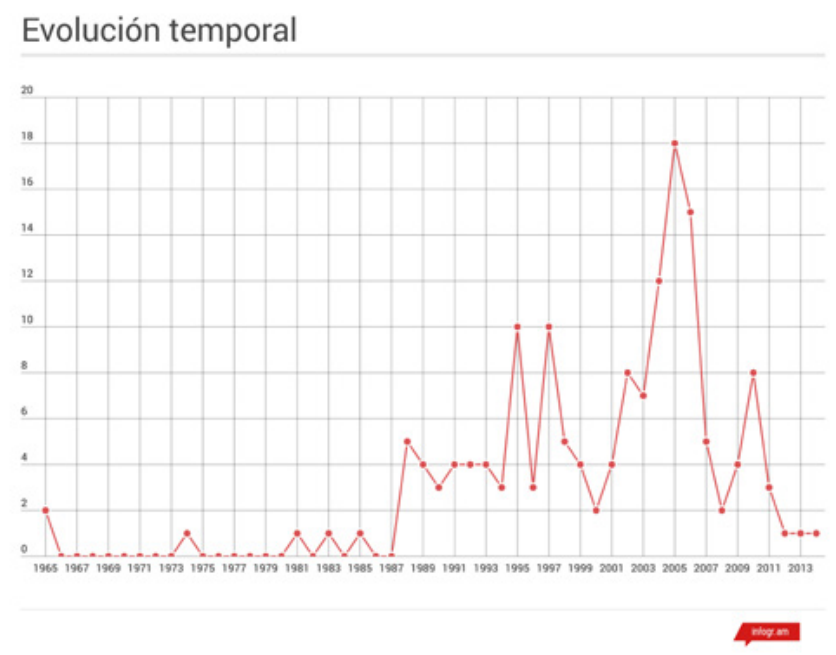

Figura 1: Evolución temporal de las referencias al concepto de hipertextualidad | Fuente: elaboración propia 
Refiniendo la hipertextualidad. Análisis cuantitativo y cualitativo de la evolución... 54

INNOVACIÓN TEÓRICA

Todo empezó en 1965. Theodor Nelson introdujo la palabra "hypertext" con una definición eminentemente abstracta, pero con importantes dosis descriptivas y comparaciones con los elementos hasta el momento conocidos. Pese a los años transcurridos y a su visión utópica, poco se puede corregir medio siglo después:

Let me introduce the word "hypertext" to mean a body of written or pictorial material interconnected in such a complex way that it could not conveniently be presented or represented on paper. It may contain summaries, or maps of its contents and their interrelations; it may contain annotations, additions and footnotes from scholars who have examined it. Let me suggest that such an object and system, properly designed and administered, could have great potential for education, increasing the student's range of choices, his sense of freedom, his motivation, and his intellectual grasp. Such a system could grow indefinitely, gradually including more and more of the world's written knowledge. However, its internal file structure would have to be built to accept growth, change and complex informational arrangements (Nelson, 1965: 96).

Ese mismo año, el propio Nelson (1965: 98) completaba esta propuesta con una aportación etimológica:

The sense of "hyper-" used here connotes extension and generality (...) The criterion for this prefix is the inability of these objects to be comprised sensibly into linear media, like the text string, or even media of somewhat higher complexity.

Casi una década después, el el propio Nelson y Brand (1974) introdujeron otro de los elementos clave de este concepto: la interacción del autor:

Means forms of writing which branch or perform on request; they are best presented on computer display screens (...) In ordinary writing the author may break sequence for footnotes or insets, but the use of print on paper makes some basic sequence essential. The computer display screen, however, permits footnotes on footnotes on footnotes, and pathways of any structure the author wants to create. Discrete, or chunk style, hypertexts consist of separate pieces of text connected by links (Nelson y Brand, 1974). 


\section{INNOVACIÓN TEÓRICA}

Siete años más tarde, el filósofo e informático presentaba las claves concretas del sistema al que estaba dando forma: "Because links exist in all kind of writings, we have endeavored to create a system for editing and retrieving documents with links, to mirror, and replicate, and extend, existing literary structure" (Nelson, 1981). Y dos años después, de nuevo Nelson (1983: 3) incidía en su naturaleza multimedia: "Text, graphics, audio and video [that] can now come alive in unified, responding, explorable new works that present facts and ideas".

Estas definiciones incluyen casi la totalidad de lo que después se ha dicho, con otras palabras y enfoques, sobre la hipertextualidad. En cierto modo, se podría afirmar que es posible entender completamente este concepto -incluso quizás con menor confusión- si no se hubiese escrito nada más al respecto. Pero el fenómeno no iba a quedar ahí $\mathrm{y}$, por lo tanto, tampoco los intentos de aproximación a su naturaleza y sus efectos. Y es que las elucubraciones no habían hecho más que comenzar.

La gran expansión llegó a finales de los ochenta, coincidiendo con el desarrollo de las primeras aplicaciones prácticas de este fenómeno (Nielsen, 1995b; Moulthrop, 2003). Y se consolidó a mediados de los noventa, gracias a la implantación de la World Wide Web, la gran manifestación global -aunque imperfecta- de la hipertextualidad (Nielsen, 1999a: 71; Rivett, 2000: 53; Hayles, 2008: 613). En el segundo lustro de los ochenta, se formularon 12 propuestas (un 7,74\% del total). Esta cifra se duplicó en la primera mitad de los noventa $(25$, un $16,13 \%)$ y se mantuvo en la segunda parte de esta década (24, un 15,48\%).

Entre las propuestas de ese primer momento de auge, destaca la de Smith y Weiss (1988), donde ya aparecen elementos clave como la red, el nodo, el enlace y la interactividad:

A form of electronic document (...) an approach to information management in which data is stored in a network of nodes and links. It is viewed through interactive browsers and manipulated through a structure editor. 
Refiniendo la hipertextualidad. Análisis cuantitativo y cualitativo de la evolución... 56

INNOVACIÓN TEÓRICA

En los noventa, merece la pena subrayar la aportación de Conklin (1995: 2021), que profundizaba en la visión de la hipertextualidad desde múltiples puntos de vista:

Windows on the screen are associated with objects in a data base, and links are provided between these objects, both graphically (...) and in the data base (...) From a computer science viewpoint the essence of hypertext is precisely that it is a hybrid that cuts across traditional boundaries. Hypertext is a database method, providing a novel way of directly accessing data which is quite different from the traditional use of queries. At the same time, hypertext as a representation scheme is a kind of "semantic network" which mixes informal textual material with more formal and mechanized operations and processes. Thirdly, hypertext is an interface modality that features "control buttons" (link icons) which may be arbitrarily embedded within the content material by the user. These are not separate applications of hypertext - they are metaphors for a functionality that is an essential union of all three.

La Figura 1 también muestra cómo, entre 1998 y 2001, se produjo lo que se podría denominar la primera "crisis" en las definiciones de este término. En 2002, en cambio, se propusieron 8 definiciones $(5,16 \%)$, y el número siguió creciendo en $2004(12$, un $7,74 \%)$ y, sobre todo, en 2005, cuando se alcanzó la cifra más elevada (18, un 11,61\%). En total, en el primer lustro del nuevo siglo, se realizaron 49 menciones al concepto de hipertextualidad (un 31,61\%), casi un tercio del total.

Entre las múltiples propuestas de ese momento, quizás la más completa sea la de Campàs (2005: 45), que aunaba las explicaciones descriptivas y abstractas:

Una tecnología que organiza una base de información en bloques discretos de contenido, llamados nodos, conectados por medio de una serie de enlaces, cuya selección que provoca la recuperación inmediata de la información de destino (...) el texto que, visualizado en un espacio tridimensional, está formado por una serie de planos que se cortan en todos aquellos puntos que representan una relación entre los conceptos que incluyen. 


\section{INNOVACIÓN TEÓRICA}

Esta tendencia se mantuvo en 2006 (15, un 9,68\%), pero a partir de 2007 descendió considerablemente (5, un 3,23\%). En 2010 se produjo un repunte (8, un $5,16 \%)$, pero el número de definiciones retrocedió a sus índices más bajos en los primeros años de esta década. La última definición localizada es la de Doherty (2014: 125), que regresaba a los orígenes e incidía en sus componentes esenciales (nodos y enlaces): “In its basic form, a hypertext consists of nodes of information connected by links. It is this link-and-node concept that provides the underlying structure of the World Wide Web".

\subsection{Tipología de las referencias}

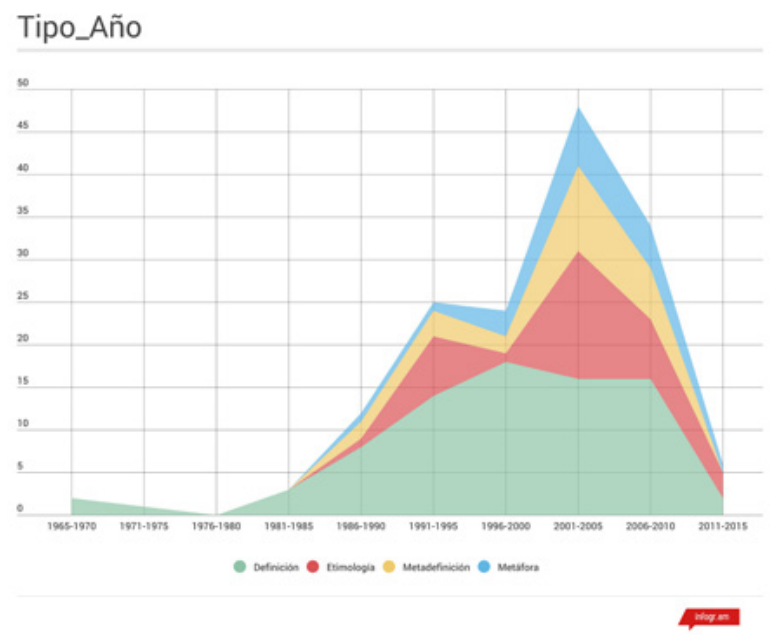

Figura 2: Tipología de las menciones al concepto de hipertextualidad | Fuente: elaboración propia

Entre las referencias sobre la hipertextualidad formuladas durante casi medio siglo, las más comunes son las definiciones propiamente dichas (80, un 51,61\%), más de la mitad del total. Esta tipología fue la única empleada durante las primeras décadas de existencia de este término. Incluso en el segundo lustro de los noventa (18, un $75 \%$ del conjunto de ese periodo) o en la primera década de este siglo (16, un $47,06 \%$ ), las definiciones alcanzan cifras considerables.

Ya se han destacado varias propuestas de este tipo, pero la pena subrayar la propuesta de Rivera (2010: 16) por su capacidad para incluir gran parte de los ele- 
mentos esenciales: “Una estructura de base informática que hace posible, a través de enlaces, la conexión electrónica de unidades textuales (dentro del texto o con otros documentos) y que requiere la manipulación activa del lector".

También poseen un peso reseñable las referencias a la etimología de este término (34, un 21,94\% del total). Este tipo de análisis presenta unas cifras reducidas a finales de los noventa ( 1 , un $4,17 \%$ del conjunto de ese periodo), pero adquiere fuerza a principios de esa década (7, un $28 \%$ ) y en el primer (7, un 20,59\%) y el segundo lustro (3, un 50\%) del nuevo siglo.

Entre este tipo de propuestas, aunque no se analice en profundidad, predominan las reflexiones sobre el concepto de "texto" (24, un 70,59\% del total), por encima de aquellas sobre el prefijo "hiper" (8, un 23,53\%) u otros como "ciber" (2, un $5,88 \%$ ). En este último caso, hay que precisar que sólo se tuvieron en cuenta aquellas menciones directamente relacionadas con la hipertextualidad. Entre las propuestas sobre el prefijo, conviene resaltar la aportación de Díaz Noci y Salaverría (2003: 86), que señalaban que proviene del griego “hyper: más allá de, sobre, encima de, exageración, pasar por encima o transposición". Al hablar sobre el núcleo de este término, Martínez Rodrigo recuerda su origen en el término textere, entretejer o trenzar, que se puede aplicar a palabras, fragmentos o bloques de textos. En este sentido, merece la pena recoger la digresión de Pérez Marco (2003: 121) sobre la innecesaria diferenciación entre la hipertextualidad y la hipermedialidad:

La multimedialidad es una característica del hipertexto como estructura textual, y (...) es ésta la que es o no multimedia. De ahí que consideramos que sólo oscurece las cosas hablar de hipermedia como un hipertexto multimedia. Preferimos considerar que el hipertexto es siempre, o puede ser, multimedia: agrupación de diferentes formatos digitalizados que se visualizan a través del ordenador. Y puesto que en la práctica, no existen hipertextos que no utilicen la posibilidad de integración de varios medios para crear sus contenidos, nos parece redundante hablar de hipermedia e hipertexto, cuando en esencia nos estamos refiriendo a la misma realidad. En todo caso, un hipertexto formado sólo de texto (lo cual no deja de ser extraño), lo que realmente demostraría es que no está utilizando todas las posibilidades inherentes al medio, sino simple- 


\section{INNOVACIÓN TEÓRICA}

mente trasladando determinada estructura impresa a otro soporte de visualización, el ordenador, que en su esencia contiene la posibilidad de la combinación de medios diversos para crear un mensaje.

Las metadefiniciones, reflexiones o recopilaciones sobre las definiciones de la hipertextualidad, presentan resultados algo más modestos (23, un 14,84\% del total), como se observa en la Figura 2. Su presencia a lo largo del tiempo se mantiene relativamente constante, y aumenta a principios de la pasada década (10, un 20,83\%).

En esta tipología, existe un considerable equilibrio entre las críticas a los excesos o los defectos en las definiciones (12, un 52,17\% del total) y las recopilaciones de estas propuestas (11, un 47,83\%). Entre las primeras, resalta la postura de Aarseth (2003: 69), que lamentaba su utilización desmesurada y no siempre honesta en parte de la producción académica:

Perhaps hypertext is just a word, one (dominant) name for the highly contested and diverse field of digital textual communication? A rhetorical surgery for a group of researchers to take control of a field? Rather than a technology or even a theoretical object, it seems to be a hyped, enthusiastic vision intended to produce or inspire a technology that will make a "better" tool for writing and reading.

Entre las segundas, habría que destacar el análisis de Rueda (2010: 179), que diferenciaba entre su tratamiento en el campo literario, donde el hipertexto se observa como "una forma de escribir alternativa a los textos convencionales", del informático, donde se reconoce como "dispositivo tecnológico (software) que permite la interacción entre nodos de información de diversa índole: textuales, gráficos, videos, sonidos".

Finalmente, la metáfora constituye el recurso menos frecuente para acercarse al concepto de hipertextualidad (18, un 11,61\% del total). Este recurso apenas se empleó en los primeros años de existencia del término, a finales de los ochenta (1, un $8,33 \%$ ) y a principios de los noventa (1, un 4\%). Su uso, en cambio, adquirió cierta relevancia a principios (7, un $14,58 \%$ ) y a finales $(5$, un $14,71 \%$ ) de la pasada década. 
Entre estas propuestas, destacan las comparaciones con elementos espaciales (6, un 33,33\%), como la de Sarlo (1997: 52) al caracterizarlo como "una esfera cuyo centro está en todas partes y su circunferencia en ninguna (...) atravesada por cientos de caminos que pueden conducir a un lector desde (...) un trozo de escritura hasta otro". También son frecuentes las asimilaciones con la neurología (5, un 27,78\%), como la que planteó Piscitelli (2005: 28-29) al relacionar la hipertextualidad con los fractales, objetos geométricos cuya estructura básica, fragmentada 0 irregular, se repite a diferentes escalas en la naturaleza. Y por último, la alusión a la composición textil (3, un 16,67\%), generalmente por las referencias al término "texto" y a su carácter reticular:

Si hay alguna imagen que permita visualizar la idea de lo que es un hipertexto, esa imagen es la de una madeja textual. En efecto, el hipertexto es un artefacto intelectualmente seductor que comporta también un reverso -en el sentido de que no nos muestra lo que esconde hasta que no hemos actuado y hemos hecho una elección. Un instrumento que puede parecer peligroso, incluso amenazante por opaco $\mathrm{y}$, sin embargo, que hay que tener presente por su especial relevancia $\mathrm{y}$ trascendencia en esta revolución textual (Borràs, 2005: 33).

\subsection{Tipos de definiciones}

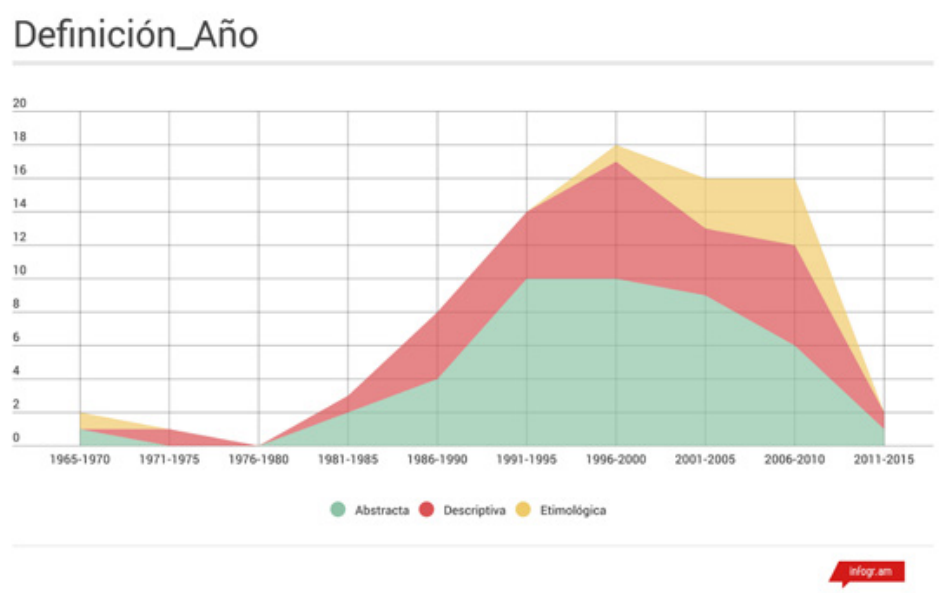

Figura 3: Naturaleza de las definiciones sobre la hipertextualidad | Fuente: elaboración propia 


\section{INNOVACIÓN TEÓRICA}

Al profundizar en las definiciones específicas de la hipertextualidad, se observa que la mayor parte adquieren un cariz abstracto (43, un 53\% del total). Del mismo modo, las descriptivas (28, un 35\%) resultan más comunes que las que se basan en la etimología (9, un 11,25\%).

Los tres tipos de definiciones presentan unas cifras bastante similares en la primera década de la existencia del término, pero a partir de los años ochenta se distancian. La abstracción predomina entre las escasas propuestas de principios de los años ochenta (2, un $66,67 \%$ del conjunto de este periodo), pero también supera claramente al resto a principios (10, un $71,43 \%)$ y a finales de los noventa (10, un $55,56 \%$ ), así como en el primer lustro del nuevo siglo (9, un 56,25\%). Entre estas propuestas, además de las ya mencionadas, conviene resaltar la de Coover (1997), que se centraba en la ruptura de la narración y la jerarquía clásica:

Es tan radicalmente nuevo que es difícil estar seguro de lo que es. Para empezar, no tiene un centro fijo y tampoco bordes, finales ni límites. La línea de tiempo narrativo tradicional desaparece en un paisaje geográfico o laberinto sin salida, en el que los comienzos, las partes centrales y los finales ya no forman parte de la visualización inmediata. En su lugar aparecen opciones que se bifurcan, menús, marcadores de vínculos y redes de mapas. No existen jerarquías en estas redes sin extremo superior (ni extremo inferior).

Las referencias más descriptivas presentaban un mayor peso relativo a finales de los ochenta (4, un 50\%\%), de los noventa (7, un 38,89\%) y de la primera década del siglo XXI (6, un 37,5\%\%). Una de las definiciones más relevantes de este tipo fue la de David Bolter (2001), uno de los grandes teóricos del lenguaje digital, que acotaba este fenómeno al considerar la hipertextualidad como "un conjunto de temas y sus conexiones, donde los primeros pueden ser párrafos, frases, trabajos individuales o incluso gráficos digitalizados". El concepto se encogía, pero a cambio incluía dos de sus elementos esenciales, el tema (o nodo) y la conexión (o enlace), y detallaba las múltiples formas que puede adoptar el primero.

Las definiciones basadas en la etimología, en cambio, no adquirieron una presencia relevante hasta el primer (3, un 18,75\%) y el segundo lustro (4, un $25 \%$ ) del 
nuevo milenio. Entre este tipo de menciones, además de las nombradas anteriormente, hay que destacar la formulada por Salaverría (2005: 29), que analizaba la nueva dimensión que le otorga su prefijo: “Un hipertexto es, según su etimología, un texto que va más allá de lo que aparenta, que se trasciende a sí mismo (...) que a la anchura y la altura propias de la página impresa suma una tercera dimensión: la profundidad".

\subsection{Elementos esenciales de las definiciones}

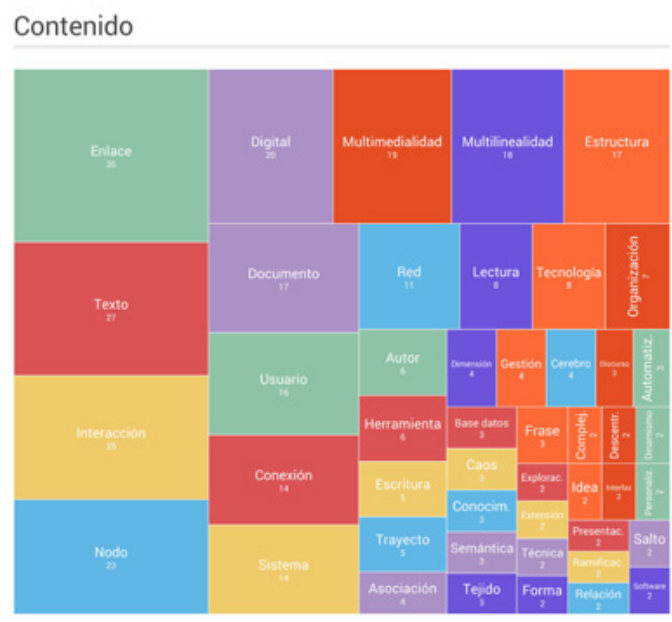

Figura 4: Contenidos de las definiciones sobre hipertextualidad | Fuente: elaboración propia

La visión más precisa de la esencia de las definiciones sobre la hipertextualidad se obtiene al clasificar los conceptos empleados. La Figura 4, que recoge sólo los que se repiten al menos dos veces, muestra cómo el término "enlace" constituye, con diferencia, el más habitual (35, un 7,66\% del total). Aunque claramente por debajo, aparecen tres palabras que complementan muchas de las propuestas estudiadas: "texto" (27, un 5,91\%), "interacción" (25, un 5,47\%) -reforzada por el uso del concepto "usuario" (16, un 3,50\%)- y "nodo" (23, un 5,03\%). No obstante, en ocasiones también se utilizan términos como "fragmento", "pieza" o "lexía", aunque aquí se ha optado por agruparlos. Y a éstos se les podría sumar otro bastante frecuente: "documento" (17, un 3,72\%). 


\section{INNOVACIÓN TEÓRICA}

A continuación, se detecta el uso de dos conceptos que determinan sendos rasgos esenciales: "digital" (20, un 4,38\%) y "multimedialidad" (18, un 3,94\%). A estos habría que añadirle otros con cierta relevancia, como "conexión" (14, un $3,06 \%$ ) o "red" (11, un 2,41\%). La "multimedialidad" constituye, con diferencia, la consecuencia más resaltada sobre la hipertextualidad (19, un 4,16\%), por encima de otras como la "asociación" (4, un 0,88\%), la "automatización" (3, un 0,66\%), el "caos" (3, un 0,66\%) o el "conocimiento" (3, un 0,66\%).

Entre los términos que describen la naturaleza primaria de la hipertextualidad, destaca la utilización de "estructura" (17, un 3,72\%) y "sistema" (14, un 3,06\%), y la presencia de "tecnología" (8, un 1,75\%) y "herramienta" (6, un 1,31\%). Entre las acciones que desencadena, en cambio, sobresale el uso de la "lectura" (8, un $1,75 \%$ ) y la "escritura" (5, un 1,09\%).

Finalmente, el conjunto de otros términos que sólo se nombran una vez también alcanza unas cifras considerables (86, un 18,82\%). Entre éstos, se podrían destacar conceptos como "bifurcación", "clic", "anclaje" o "pliegue".

\section{Conclusiones}

La hipertextualidad, vestigio de la prehistoria de la documentación y la intertextualidad, nudo de las autopistas de la información, eje de los primeros mecanismos digitales de atribución y construcción colaborativa y botón de acción en los relatos interactivos e inmersivos. Un término con más de medio siglo de historia, decenas de detractores y entusiastas. Y sin embargo, con tantas incógnitas que resolver.

Tras la iluminación de un alquimista de la informática y la filosofía como Theodor Nelson, las propuestas sobre este término permanecieron en el olvido durante más de dos décadas. La popularización de la informática, a finales de los ochenta, y sobre todo la explosión de la Web, en los noventa, convirtieron la hipertextualidad en un concepto de moda. Tanto, que las primeras propuestas teóricas, etimológicas y metafóricas pronto se complementaron con visiones mucho más críticas sobre los excesos y la banalización de su utilización. 
Pero los resultados de esta investigación también demuestran que, a pesar de lo que a priori se podría intuir, la mayor parte de las menciones expresas a la hipertextualidad llegaron a partir del año 2000. Probablemente, cuando una parte mucho mayor de los usuarios -y entre ellos, los periodistas- tuvieron la oportunidad o la necesidad de ponerla en práctica ante el auge de la Web 2.0. En este periodo, las definiciones clásicas incluso perdieron protagonismo ante intentos como las metáforas o las propuestas metodológicas, quizás en un intento de hacer más comprensible este concepto.

Esta tendencia coincide con la disminución de las definiciones abstractas en favor de las más descriptivas y etimológicas. Ante la necesidad de usar esta estructura, convenía dejar a un lado las digresiones filosóficas. Había llegado el momento de enumerar sus componentes, explicar su naturaleza o su funcionamiento y plantear sus implicaciones en la escritura y la lectura.

Pero los elementos esenciales siempre habían estado ahí. El enlace conforma el eje de la hipertextualidad. Eso se refleja en sus revisiones teóricas, en las que también se hace referencia a conceptos relacionados como la conexión o la red. Estos enlaces sólo pueden servir para vincular contenidos, que en las definiciones se identifican como textos, nodos, elementos multimedia o documentos. Para que se produzca esta conexión, siempre se requiere la interacción del usuario, además de su desarrollo y presentación en un entorno digital. La consecuencia más relevante de sus decisiones es la multilinealidad. Y al concepto que lo engloba todo se le podría llamar estructura o sistema.

A partir de todo esto, la propuesta más adecuada a sus antecedentes para definir la hipertextualidad podría ser la siguiente:

Una estructura construida a partir de los enlaces que unen textos o contenidos multimedia mediante la interacción del usuario en un entorno digital y multilineal.

Esta definición incluye todos los elementos esenciales de este concepto con sus formulaciones más frecuentes. La hipertextualidad puede concebirse, en efecto, como 


\section{INNOVACIÓN TEÓRICA}

una estructura, con las referencias que este término posee sobre la relación entre sus partes o su vinculación con acciones como la construcción. El enlace, sin duda, constituye el núcleo básico de un objeto que, aunque no sea necesario precisarlo, puede estar compuesto por palabras o imágenes fijas o en movimiento. La participación del usuario, finalmente, resulta imprescindible para desplegar unos contenidos que siempre se despliegan en algún tipo de interfaz informática para dar lugar a un recorrido multilineal. No obstante, si se eliminan los elementos comprensibles por sí mismos o por el contexto, esta definición se podría simplificar de este modo:

La conexión de contenidos mediante enlaces activados por el usuario.

Sin duda, ni esta definición ni el conjunto del trabajo carecen de limitaciones. En el proceso de búsqueda $\mathrm{y}$, sobre todo, en el de preselección, es posible que se hayan ignorado trabajos relevantes. Sobre todo, por la influencia del factor humano cuando estas referencias se apartaban de los canales más habituales y por la exclusión de las propuestas realizadas en idiomas distintos al castellano o al inglés. Del mismo modo, el proceso de análisis también se podría haber perfeccionado con un mayor aprovechamiento del software de análisis cualitativo de contenido existente.

No obstante, este artículo permite arrojar algo de luz sobre un concepto tan controvertido como la hipertextualidad mediante técnicas como la recopilación, la

sistematización y el análisis cuantitativo y cualitativo. De este modo, a partir del estudio de la evolución de sus tendencias, elementos comunes y matices, se espera haber clarificado el significado y el alcance de un término con tanto recorrido como horizonte por delante. Como muchas otras palabras, probablemente su destino sea la desaparición, pero mientras tanto conviene conocer su pasado y destilar su significado para entenderla $\mathrm{y}$, sobre todo, aplicarla con precisión.

\section{Bibliografía}

Aarseth, E. J. (2003). Nonlinearity and literary theory. En Landow, G. P. (1994). Hyper/Text/Theory, 51-86. Baltimore: Johns Hopkins University Press. 
Refiniendo la hipertextualidad. Análisis cuantitativo y cualitativo de la evolución... 66

INNOVACIÓN TEÓRICA

Andréu, J. (2001): Las tecnicas de Analisis de Contenido: Una revisión actualizada. Centro de Estudios Andaluces. Recuperado de: http://public. centrodeestudiosandaluces.es/pdfs/S200103.pdf.

Bolter, J. D. (2001). Writing space: Computers, hypertext, and the remediation of print. Kentucky: Routledge.

Borràs, L. (ed.) (2005). Teorías literarias y retos digitales. En Borràs, L. (ed.). Textualidades electrónicas. Nuevos escenarios para la literatura. Barcelona: Editorial UOC.

Cáceres, P. (2008). Análisis cualitativo de contenido: Una alternativa metodológica alcanzable. Psicoperspectivas, 2 (1), 53-82. Recuperado de: http://www. psicoperspectivas.cl/index.php/psicoperspectivas/article/viewFile/3/3

Campàs, J. (2005a). L'hipertext. Barcelona: Editorial UOC.

Clauso, A. (1993). Análisis documental: el análisis formal. Revista general de información y documentación, 3 (1), 11-19. Recuperado de: http://revistas. ucm.es/index.php/RGID/article/viewFile/RGID9393120011A/11739

Conklin, J. (1995). A survey of hypertext. En ACM's Hypertext on Hypertext HyperCard. Recuperado de: http://citeseerx.ist.psu.edu/viewdoc/download?d oi=10.1.1.41.9368\&rep=rep1\&type=pdf

Coover, R. (1992). The end of books. New York Times Book Review, 21 (6), 23-25. Recuperado de: https://www.nytimes.com/books/98/09/27/specials/cooverend.html

Doherty, S. (2014). Hypertext and Journalism: Paths for future research. Digital Journalism, 2 (2), 124-139.

Dulzaides, M. E. y Molina, A. M. (2004). Análisis documental y de información: dos componentes de un mismo proceso. Acimed, 12 (2), Recuperado de: http://scielo. sld.cu/scielo.php?script=sci_arttext\&pid=S1024-94352004000200011\&lng=es \&tlng=es.

Hayles, N. K. (2008). Electronic literature: new horizons for the literary. París: University of Notre Dame Press.

Krippendorff, K. (1990). Metodología de análisis de contenido: teoría y práctica. Barcelona: Paidós.

Martínez Rodrigo, E. Lectores dinámicos ante textos interactivos. Icono 14. Revista científica de Comunicación y Tecnologías emergentes, 8 (1), 261-273. Recuperado de: http://www.icono14.net/ojs/index.php/icono14/article/view/293. 


\section{INNOVACIÓN TEÓRICA}

Mendoza, M. A. (2000). Análisis de contenido cualitativo y cuantitativo: definición, clasificación y metodología. Revista de Ciencias Humanas, 20. Recuperado de: http://www.utp.edu.co/ chumanas/revistas/revistas/rev20/gomez.htm

Moreno, I. (2002). Musas y nuevas tecnologías: el relato hipermedia. Barcelona: Paidós.

Moulthrop, S. (2003). El hipertexto y la política de la interpretación. En J. Vega, M. (ed.). Literatura hipertextual y teoría literaria, 23-69. Madrid: Mare Nostrum Comunicación.

Nelson, T. H. (1965). Complex information processing: a file structure for the complex, the changing and the indeterminate. En 20th national conference, 84-100. Recuperado de: http://rogerclarke.com/II/Nelson-1965.pdf

Nelson, T. H. (1981). Literary machines. Berkeley: Mindful Pr.

Nelson, T. H. (1989). Hyperwelcome. Hypermedia, 1 (1), 3-5.

Nelson, T. H. y Brand, S. (1974). Computer Lib: You can and must understand computers now. Washington: Tempus Books/Microsoft Press

Nielsen, J. (1995a). Multimedia and hypertext: The Internet and beyond. 0xford: Morgan Kaufmann.

Nielsen, J. (1995b). The History of Hypertext. Nielsen Norman Group. Recuperado de: http://www.nngroup.com/articles/hypertext-history/

Peña, T. y Pirela, J. (2007). La complejidad del análisis documental. Información, Cultura y Sociedad, 16, 55-81. Recuperado de: http://eprints.rclis.org/17138/

Pérez Marco, S. (2003). El concepto de hipertexto en el periodismo digital: análisis de la aplicación del hipertexto en la estructuración de las noticias de las ediciones digitales de tres periódicos españoles (www. elpaís. es, www. elmundo. es, www. abc. es) [Tesis doctoral]. Universidad Complutense de Madrid. Facultad de Ciencias de la Información.

Piscitelli, A. (2005). Internet: la imprenta del siglo XXI. Barcelona: Gedisa.

Rivera, Y. (2010). El hipertexto y la polifonía del sentido. Educarambar. Recuperado de: http://educarambar.blogspot.com.es/2010/11/el-hipertexto-y-la-polifoniadel.html

Rivett, M. (2000). Approaches to Analysing the Web Text: A Consideration of the Web Site as an Emergent Cultural Form. Convergence: The International Journal of Research into New Media Technologies, 6 (3), 34-56. doi: 10.1177/135485650000600304 
Rueda, R. (2010). Formación, hipertexto y ambientes de aprendizaje. Revista Educación y Pedagogía, 7 (14), 179-196. Recuperado de: http://aprendeenlinea. udea.edu.co/revistas/index.php/re-vistaeyp/article/viewFile/5587/5009

Salaverría, R. (2005). Hipertexto periodístico: mito y realidad. Tripodos, 67 (1), 517-524. Recuperado de: http://dadun.unav.edu/bitstream/10171/5095/1/ tripodos2005_hipertexto_perio-distico.pdf

Sarlo, B. (1997). Del plano a la esfera: libros e hipertextos. Palabra Clave, 2, 47-53. Recuperado de: http://palabraclave.unisabana.edu.co/index.php/ palabraclave/article/download/339/481

Smith, J. B., \& Weiss, S. F. (1988). An overview of hypertext. En Communications of the ACM, 887-895. Recuperado de: http://dl.acm.org/citation.cfm?id=48512 